\title{
Location and Volume Determination of Hydrogen Refueling Stations Based on Oligopoly Equilibrium
}

\author{
Yuansheng Huang ${ }^{1}$, Lixia Tian ${ }^{1}$, Hengfeng Zhao $^{2}$, Shize Sun ${ }^{1}$, Jiajia Deng ${ }^{3}$, Shuang Liu ${ }^{4 *}$ \\ ${ }^{1}$ Department of Economy and Management, North China Electric Power University, Baoding 071002, China \\ ${ }^{2}$ PetroChina North China Petrochemical Company, Renqiu 071002, China \\ ${ }^{3}$ State Grid Communication Company, Beijing 100052, China \\ ${ }^{4}$ College of Quality and Technical Supervision, Hebei University, Baoding 071002, China
}

Corresponding Author Email: liushuang99@hotmail.com

https://doi.org/10.18280/ijht.390110

Received: 17 October 2020

Accepted: 26 December 2020

\section{Keywords: \\ hydrogen fuel cell vehicles (HFCVs), hydrogen refueling stations (HRSs), Multi- agent optimization problem with equilibrium constraint (MOPEC), market equilibrium, oligopoly equilibrium}

\begin{abstract}
The energy transformation in China is picking up speed, thanks to the mass development and utilization of various clean energies. As an emerging clean energy, hydrogen energy has been highly recognized, and gradually introduced to industry and transport. As a result, hydrogen fuel cell vehicles (HFCVs) attract a growing attention from the government and the public. However, the is a severe lack of hydrogen refueling stations (HRSs). Therefore, this paper explores into the determination of HRS location and volume. The HRS investment market was treated as an oligopoly market, due to the huge investment and few investors of HRSs. To ensure the long-term balanced development of hydrogenation, it is assumed that all investors fully analyze the possible investment behaviors of competitors and HFCV driver preference before making any investment decision. On this basis, a multiagent optimization model was established to determine the location and volume of HRSs. The results show that, to avoid supply-demand imbalance induced by information asymmetry, the HRSs should be constructed along with an intelligent software platform, which provides HFCV drivers with rich information on hydrogenation service. Our model helps investors determine the core issues of the HRS, including location, size, and supply scale.
\end{abstract}

\section{INTRODUCTION}

Energy is a main driver of social progress and technological advancement. In recent years, the energy demand continues to increase around the world. However, the traditional fossil energy is limited in supply, and a major source of air pollutants. To bridge the demand-supply gap, clean energies have developed rapidly on the global scale.

One of the most popular clean energies is hydrogen energy [1], which is virtually inexhaustible. Hydrogen is ubiquitous in nature. Most of hydrogen exists in the form of compound water. About $70 \%$ of the Earth's surface is covered by water, $11 \%$ of which is hydrogen. Thus, the Earth itself is a huge warehouse of hydrogen. Hence, hydrogen energy is hailed as the main energy of the new century.

Currently, hydrogen fuel cell vehicles (HFCVs) attract a growing attention from the government and the public [2, 3]. hydrogen refueling station (HRS) is a necessary infrastructure of HFCVs [4]. With the growing number of HFCVs [5], the HRS market has been gradually opened [6]. China had constructed 76 HRSs by September 2020, and there are 10 HRSs under construction and 78 in the planning stage [7].

Many experts and scholars have probed deep into HFCVs and HRSs. Some have demonstrated the competitiveness of hydrogen energy and HFCVs in future market from the perspective of cost [8] and environment [9]. For example, Liu et al. [10] calculated the investment cost and operation cost of HRSs. Shan et al. [11] computed the overall cost of hydrogen energy supply chain, including edges like hydrogen production, transportation, and hydrogenation.

Some have discussed the hydrogen safety of HFCVs and HRSs. For instance, Dai and Zhang [12], Shi et al. [13] measured the level of safety of HRRs. Zheng [14], Zheng et al. [15] evaluated the safety of hydrogen storage devices, as well as that of hydrogen transportation process.

Some have studied the design and layout of HRSs. Agll, Agll et al. [16] designed a novel embedded HRS. Liu [17] controlled the HRS construction of HRS from the angle of project management. André et al. [18], Ramea [19] located hydrogen pipelines and examined the mode of hydrogen supply.

Some have identified the influencing factors and satisfaction of HRS selection among HFCV drivers [20, 21]. Kelley et al. [21] pointed out that the drivers' choice of HRS depends greatly on subjective and objective convenience.

Some have determined the location and volume of HRSs. Thiel [22] considered distance and price as two main reasons for drivers to choose HRSs, and proposed a two-step pricingbased location strategy. Gao et al. [23], Li et al. [24], He et al. [25], Sun [26] selected HRS sites according to the overall cost of hydrogen supply chain. Lin et al. [27] integrated data from multiple sources to the HRS location model, including gas station network, geographic information system (GIS), population, and regional economy. Miralinaghi et al. [28] located HRSs in the light of driving route selection and hydrogenation demand uncertainty. Lewandowska- 
Śmierzchalska et al. [29], Narayanamoorthy et al. [30], Deveci [31] relied on analytic hierarchy process (AHP) and fuzzy set to locate underground hydrogen storage tanks. Yang and Jiang [32] fully considered operation cost and demand uncertainty in an attempt to determine the location and volume of HRSs. Grassman et al. [33] designed the HRS volume based on consumer satisfaction.

The determination of HRS location and volume is essentially an oligopoly game. For the long-term balanced development of hydrogenation market, this paper explores the determination of HRS location and volume based on oligopoly equilibrium, and establishes a multi-agent optimization model to help investors solve this problem. The research was carried out under two assumptions: all investors enter the market at the same time; every investor fully analyzes the investment behavior of the current and potential competitors before making its own decision on investment.

\section{MODELING}

The main purpose of this paper is to establish an HRS network that facilitates the oligopoly equilibrium of the hydrogenation market, allocates resources reasonably, and satisfies the long-term demand of hydrogenation. Before modeling, it is assumed that all investors enter the market at the same time; every investor fully analyzes the investment behavior of other investors, before making its own decision on investment.

The HRS market is not large at present. But the market scale will continue to expand, with the growing number of HFCVs. Many new investors will be attracted to the market. Therefore, any investment decision should be made in view of the investment behavior of the existing investors, as well as that of potential entrants to the market.

Under the condition of market equilibrium, the hydrogenation price of HRSs is completely determined by the market. However, the hydrogenation cost varies with locations, due to traffic congestion, and accessibility of the traffic network. Thus, the hydrogenation price would change from place to place. This paper assumes that hydrogenation demand of HFCVs depends only on traffic conditions and hydrogenation services.

On this basis, a multi-agent optimization problem with equilibrium constraint (MOPEC) model was established to analyze the interaction between investors.

\subsection{MOPEC modeling framework}

In 2012, Ferris and Wets proposed a general modeling framework for MOPEC [34]. Since then, this framework has been repeatedly theorized and applied [35-38]. Here, the basic MOPEC model is expressed as:

$$
x_{i} \in \arg \max _{x} f_{i}\left(\partial, x, x_{-i}\right), \quad i \in I
$$

where, $I$ is the set of investors; $x_{I}=\left(x_{i}, i \in I\right)$ is the investment behavior; $f_{i}$ is the criterion function of investment behavior; $x_{-i}$ is the investment behavior of investors other than investor $\mathrm{i}$; $\partial$ is a parameter, whose relationship with $x_{I}$ satisfies the market equilibrium constraints.

\subsection{Investment behavior of each investor}

\subsubsection{Behavior modeling}

After deciding to enter the market, an investor needs to make three decisions: (1) where to build the HRS? (2) what is the scale of the HRS? (3) how many hydrogenation services should the HRS provide to the market during the operation phase. The first two investment behaviors occur in the planning phase, before the investor enters the market; the third behavior occurs in the operation phase, after the investor enters the market.

Every decision made by an investor is based on the investment behaviors of other investors and potential entrants:

$$
\begin{array}{cc}
\underset{v_{i}^{l}, s_{i}^{l}}{\max } \operatorname{imize}_{l \in L_{i}}\left[p^{l} s_{i}^{l}-\varphi_{s}\left(\mathbf{s}^{\mathbf{l}}\right)\right]-\sum_{l \in L_{i}} \varphi_{v}\left(v_{i}^{l}\right) \\
\text { Subject to } \quad\left(\gamma_{i}^{l}\right) \quad s_{i}^{l}-v_{i}^{l} T \leq 0, \forall l \in L_{i} \\
s_{i}^{l} \geq 0, \forall l \in L_{i} \\
v_{i}^{l} \geq 0, \forall l \in L_{i}
\end{array}
$$

where, $L_{i}$ is the set of candidate locations selected by investor $i, \forall l \in L_{i} ; v_{i}^{l}$ is the volume of the HRS established by investor $i$ at location $l$ (unit: $\mathrm{kg}$ ); $T$ is the duration of hydrogenation in peak hours; $s_{i}^{l}$ is the amount of hydrogen provided by investor $i$ at location $l$ during the peak period $T$ (unit: $\mathrm{kg}$ ); $p^{l}$ is the market price of hydrogenation service at location $l ; \varphi_{v}(\cdot)$ is total capital cost based on the total volume of hydrogen storage vessels in the HRS; $\varphi_{S}(\cdot)$ is the operation cost based on the number of hydrogenation services provided by the HRS and the operation plan; $I$ is the set of investors, $\forall i \in I ; \gamma_{i}^{l}$ is the constraint on the volume for investor $i$ at location $l$. As mentioned before, investor $i$ needs to determine three things: the station location $l$, station volume $v_{i}^{l}$, and number of hydrogenation services $s_{i}^{l}$ during operation.

The objective function (2a) aims to maximize the maximum net income, which equals the maximum net operating income minus the total investment cost. In this function, $\sum_{l \in L_{i}} p^{l} s_{i}^{l}$ is the maximum operating income of the HRS received by investors; $\sum_{l \in L_{i}}\left[p^{l} s_{i}^{l}-\varphi_{s}\left(s^{l}\right)\right]$ is the largest operating net income of the HRS received by investors; $\sum_{l \in L_{i}} \varphi_{v}\left(v_{i}^{l}\right)$ is the total investment cost of investors to establish the HRS. Note that all lowercase bold fonts represent vectors, e.g., $\boldsymbol{s}^{l}$ in $\sum_{l \in L_{i}} \varphi_{S}\left(\boldsymbol{s}^{l}\right)$ is a vector.

The total investment cost function $\varphi_{v}(\cdot)$ is a linear function, and the operation cost function $\varphi_{s}(\cdot)$ is a quadratic function with positive coefficients. Besides ensure the concavity of the objective function, this explains two micro-economic phenomena:

(1) The growing hydrogenation demand in a location will aggravate the congestion of the traffic network, pushing up the hydrogenation price at this location;

(2) The rise of hydrogenation price will attract more potential competitors to the market, and result in an oversupply of hydrogenation service. 
Under the condition of market equilibrium, the price of hydrogenation service at a location remains the same, which is determined by the market.

Constraint ( $2 b$ ) ensures that the number of hydrogenation services provided by the HRS during peak hours is smaller than or equal to the total volume of hydrogen storage vessels. Here, it is assumed that the hydrogen in the vessels can be replenished in time.

Constraints (2c) and (2d) guarantee that the relevant parameters are not negatives.

\subsubsection{Decision modeling}

Before investing in an HRS, investors must consider such factors as the station location, the volume of hydrogen storage vessels, and the hydrogenation demand at the location. The investment behavior of investors is not affected by HFCV ownership, but by the HFCV driver decisions. When an HFCV drives in the basic traffic network, the driver chooses the route based on traffic congestion and toll facilities. Hence, this paper takes HFCV ownership as an exogenous variable.

The combined distribution and assignment (CDA) model $[39,40]$ was adopted to plan the hydrogenation infrastructure. This model was adopted by Ryu et al. [41], Yao et al. [42] to solve optimization problems. The model can simulate the interaction between HFCV drivers and hydrogenation infrastructure.

Inspired by Kelley et al. [21], four factors were selected as the main influencing factors on HFCV drivers' selection of hydrogenation service, namely, location, travel time, total volume of vessels, and hydrogenation cost. Then, a multinomial logic model was established for the drivers' route selection. The deterministic components of the model's utility function are as follows:

$$
U^{k l}=\beta_{0}^{l}-\beta_{1} t^{k l}+\beta_{2} \sum_{i \in I_{l}} v_{i}^{l}-\beta_{3} \frac{p^{l} e^{k l}}{i n c^{k}}
$$

where, $K$ is the set of origins (Os) of a route, $k \in K$; $L$ is the set of destinations (Ds) of a route, $l \in L ; I_{l}$ is the set of investors who may invest in location $l, i \in I_{l} ; U^{k l}$ is the utility of a driver as he/she drives from location $k$ to location $l ; \beta$ is a parameter of the utility function (model input); $t^{k l}$ is the mean travel time from location $k$ to location $l ; e^{k l}$ is the mean hydrogenation demand from location $k$ to location $l$ (model input); $i n c^{k}$ is the mean income of locals in location $k$ (model input).

Formula (3) is the utility function of the driver whose drives from location $k$ to location $l$. According to the formula, when he/she drives an HFCV from location $k$ to location $l$, the driver's utility is affected by the location attraction, travel time, total volume of vessels, and hydrogenation cost.

Specifically, $\beta_{0}^{l}$ is the attraction of location $l$ to $\mathrm{HFCV}$ driver; $\beta_{1} t^{k l}$ is the travel time from location $k$ to location $l$ for HFCV drivers; $\beta_{2} \sum_{i \in I_{l}} v_{i}^{l}$ is the attraction of the total volume of vessels at location $l$ to HFCV drivers; $\beta_{3} \frac{p^{l} e^{k l}}{i n c^{k}}$ is the effect of hydrogenation cost on HFCV drivers driving from location $k$ to location $l$.

Among them, $\beta_{0}^{l}$ changes with the HFCV drives and locations. For example, if location $l$ is the driver's workplace, then the location attraction will play a key role in the utility function, and $\beta_{0}^{l}$ should be given a high value. By contrast, if the driver only goes to location $l$ for shopping, then going to this location is purely accidental, because different shopping malls have the same effect on the driver. In this case, the location attraction is not the key impactor of the utility function; the driver's station choice is not only affected by location attraction, but also by travel time and hydrogenation cost.

Next, the traffic network was illustrated as a directed graph $G=(N, A)$, where $N$ is the set of nodes in the network, $n \in N$; $A$ is the set of edges in the network, $a \in A$. Each edge refers to the connection between any two nodes. Then, the CDA model can be established as:

$$
\begin{aligned}
& \underset{x, f, q}{\min \operatorname{imize}_{a}} \sum_{a \in A} \int_{0}^{f_{a}} t_{a}(u) d u+\frac{1}{\beta_{1}} \sum_{k \in K} \sum_{l \in L} q^{k l}\left(\operatorname{Inq} q^{k l}-1+\beta_{3} \frac{p^{l} e^{k l}}{\operatorname{inc}^{k}}-\beta_{2} \sum_{i \in I_{+}} v_{i}^{l}-\beta_{0}\right) \\
& \text { Subject to } f_{a}=\sum_{k \in K} \sum_{l \in L} x_{a}^{k l}, \forall a \in A \\
& \left(\xi^{k l}\right) \quad A \boldsymbol{x}^{k l}=q^{k l} E^{k l}, \forall k \in K, \forall l \in L \\
& \left(\eta^{k}\right) \quad \sum_{l \in L} q^{k l}=d^{k}, \forall k \in K \\
& x_{a}^{k l} \geq 0, \forall a \in A, \forall k \in K, \forall l \in L \\
& q^{k l} \geq 0, \forall k \in K, \forall l \in L
\end{aligned}
$$

where, $f_{a}$ is the traffic flow on edge $a ; t_{a}(\cdot)$ is time function of HFCVs driving on edge $a ; q^{k l}$ is the traffic flow from location $k$ to location $l ; x_{a}^{k l}$ is the traffic flow on edge $a$ associated with OD pair $k l ; A$ is an incidence matrix of network nodes and edges (topology-based input); $E^{k l}$ is correlation vector of OD pair $k l$ (if the origin $k+1$, then the destination $l-1$; topologybased input); $d^{k}$ is the number of HFCVs leaving from location $k$ (model input); $\xi^{k l}$ is the constraint on traffic flow from location $k$ to location $l ; \eta^{k}$ is a constraint on traffic flow at location $k$.

In objective function (4a), $\sum_{a \in A} \int_{0}^{f_{a}} t_{a}(u) d u$ is the total user cost in the traditional static traffic equilibrium model; $q \operatorname{Inq} \quad$ in $\quad \frac{1}{\beta_{1}} \sum_{k \in K} \sum_{l \in L} q^{k l}\left(\operatorname{Inq} q^{k l}-1+\beta_{3} \frac{p^{l} e^{k l}}{i n c^{k}}-\right.$ $\beta_{2} \sum_{i \in I_{l}} v_{i}^{l}-\beta_{0}$ ) is the entropy of distribution of HFCVs; the other parts are the utility measurements of HFCV drivers. Constraint (4b) computes the traffic flow $f_{a}$ on aggregate edge $a$ by $x_{a}^{k l}$; constraint (4c) ensures the conservation of flow of the HFCVs at each node; constraint (4d) specifies that the total HFCVs leaving from node $k$ must be equal to the total travel demand.

\subsubsection{Market clearing condition}

To derive the market clearing price, the authors set a market clearing condition: the demand of hydrogenation service in a place equals the total supply of hydrogenation service in that place. For simplicity, HFCVs are only hydrogenated at the destination. Hence, the market clearing condition can be expressed as:

$$
\left(p^{l}\right) \quad \sum_{i \in I_{l}} s_{i}^{l}-\sum_{k \in K} e^{k l} q^{k l}=0, \forall l \in L
$$


Note that this paper only considers the HFCVs in need of hydrogenation service. To describe any other travel mode, a subscript should be added in the lower right corner of each parameter.

\section{SOLVING ALGORITHM}

It is a great challenge to solve a MOPEC in complex traffic network. Through variational analysis, this paper establishes a bivariate function, and finds the variational convergence of this function.

Firstly, the search for the equilibrium market clearing price of hydrogenation was converted into the search for the maximum value of the appropriate function. Then, the authors talked about the construction of bivariate function, and the relevant convergence theorems. Finally, the solving algorithm for MOPEC was described in details.

Specifically, the investment behavior of an investor under the maximum return was obtained from the investment behavior model (2a-2d) of investors in the HRS (Subsection 2.2.1). Further, the price $p \in R_{+}^{l}$ of hydrogenation service was derived by solving $\left(v_{i}^{l}(p), s_{i}^{l}(p)\right)_{l \in L_{i}}$. Meanwhile, the total volume of vessels in all HRSs in the location was obtained as $v^{l}(p)=\sum_{i \in I} v_{i}^{l}(p), l \in L$.

With the help of the drivers' decision model (4a-4b) (Subsection 2.2.2), the total volume $v(p)$ of vessels and the price $p$ of hydrogenation service were determined by calculating $(x(p, v(p)), f(p, v(p)), q(p, v(p)))$.

If the hydrogenation service available exceeds the demand, the excess can be solved by the following formula,

$$
E S_{l}(p)=\sum_{i \in I}\left(s_{i}^{l}(p)-\sum_{k \in K} e^{k l} q^{k l}(p, v(p))\right), l \in L
$$

According to equilibrium constraint (5), when the price of hydrogenation service is the equilibrium price $p^{*} \in R_{+}^{l}$, then $E S\left(p^{*}\right)=0$.

In this paper, Walrasian function is used to solve the above equilibrium problem:

$$
W(p, \varphi)=-\sum_{l \in L} \varphi_{l}\left(E S_{l}(p)\right)^{2}, \quad \text { on } R_{+}^{L} \times \Delta_{l}
$$

where, $\Delta_{l}$ is an L-dimensional simplex.

\section{CONCLUSIONS}

As an emerging clean energy, hydrogen energy has won more and more recognition. The government also strongly encourages the promotion and application of HFCVs. In the meantime, many have noticed the short supply of hydrogenation services. As a result, people begin to consider investing in the infrastructure of hydrogenation services.

Due to the sheer scale of the HRS, the investment market of HRSs is an oligopoly market. Admittedly, the market of hydrogenation service is still small. But the market size is expected to explode in the future. To realize the long-term stable development of this market, the authors set the equilibrium constraints on the investment planning of HFCV hydrogenation infrastructure.

The game psychology of human was fully considered to study the investment behavior of investors. It is assumed that every investor takes account of the investment behavior of current and potential investors before making any decision on investment. An investor can finalize his/her investment decision after going through several stages of the game. In addition, every investor was assumed to be fully aware of the behavior of HFCV drives in the traffic network.

Under the above assumption, an MOPEC model was chosen to solve the investment planning problem of HFCV hydrogenation infrastructure, aiming to help investors determine the core issues of the HRS, including location, size, and supply scale.

For convenience, this paper only considers the travel mode of HFCVs in the traffic network, and stipulates that all vehicles are hydrogenated only at the destination. This simplification leaves ample room for future research, because the real traffic network consists of fuel vehicles and electric vehicles, apart from HFCVs; the HFCVs can enjoy hydrogenation service at nodes other than the destination.

Currently, the application of hydrogen energy is not yet mature. The number of HFCVs and HRSs are far from enough. The resulting lack of historical data makes it impossible to carry out an actual case analysis. This problem will be solved in the follow-up study.

To avoid supply-demand imbalance induced by information asymmetry, the HRSs should be constructed along with an intelligent software platform, which provides HFCV drivers with rich information on hydrogenation service: the station location, the real-time volume of residual hydrogen in the station, and the hydrogenation price of each station. Such a platform can improve the competition in the hydrogenation industry, and make the hydrogen distribution more reasonable in each station. Then, no vehicle will go to an HRS with no hydrogen left for hydrogenation. In this way, the hydrogenation service market will reach equilibrium very quickly.

\section{ACKNOWLEDGMENT}

The work is supported by the National Natural Science Foundation of China (Grant No.: 71201057, 71471061, 71701069).

\section{REFERENCES}

[1] Ahmad, M.S., Ali, M.S., Rahim, N.A. (2021). Hydrogen energy vision 2060: Hydrogen as energy carrier in Malaysian primary energy mix - Developing P2G case. Energy Strategy Reviews, 35: 100632. https://doi.org/10.1016/J.ESR.2021.100632

[2] Chen, Y., Liu, J. (2021). The industrial development and supporting policies for hydrogen energy for vehicles in Japan. Contemporary Economy of Japan, (2): 80-94. https://doi.org/10.16123/j.cnki.issn.1000355x.2021.02.007

[3] Zhang, L., Guan, S., Hou, J., Sun, X. (2020). Global industrial policy and research trends in the field of new energy technologies. Coal Economic Research, 40(12): 70-75. https://doi.org/10.13202/j.cnki.cer.2020.12.011

[4] Olav, R.H. (2020). Hydrogen infrastructure - Efficient risk assessment and design optimization approach to ensure safe and practical solutions. Process Safety and 
Environmental Protection, 143: 164-176. https://doi.org/10.1016/j.psep.2020.06.028

[5] Wu, X.Y., Zuo, Z.L., Guo, S.Y., Wang, R., He, J.H. (2020). Evaluation on city application readiness of fuel cell logistics vehicles. Energy Storage Science and Technology, 9(5): 1574-1584. https://doi.org/10.19799/j.cnki.2095-4239.2020.0152

[6] Song, P., Sui, Y., Shan, T., Hou, J., Wang, X. (2020). Assessment of hydrogen supply solutions for hydrogen fueling station: A Shanghai case study. International Journal of Hydrogen Energy, 45(58): 32884-32898. https://doi.org/10.1016/j.ijhydene.2020.09.117

[7] Liu, J.H., Li, G.Z., Zheng, J.Y., Liu, Y.T., He, G.L., Zhou, X.R., Li, L., Chen, L. (2021). Research on classification principle of hydrogen fuelling station. Gas \& Heat, 41(1): 10-16, 39, 45. https://doi.org/10.13608/j.cnki.10004416.2021.01.015

[8] Li, Y.F., Shigeru, K. (2021). Economic competitiveness and environmental implications of hydrogen energy and fuel cell electric vehicles in ASEAN countries: The current and future scenarios. Energy Policy, 148(B): 111980. https://doi.org/10.1016/j.enpol.2020.111980

[9] Logan, K.G., Nelson, J.D., Hastings, A. (2020). Electric and hydrogen buses: Shifting from conventionally fuelled cars in the UK. Transportation Research Part D: Transport and Environment, 85: 102350. https://doi.org/10.1016/j.trd.2020.102350

[10] Liu, S.J., Ma, J.X., Zhou, W., Pan, X.M. (2006). Cost analysis for a mini-network of hydrogen refueling stations. Natural Gas Chemical Industry, 31(5): 44-48. https://doi.org/10.3969/j.issn.1001-9219.2006.05.011

[11] Shan, T.W., Song, P.F., Li, Y.W., Hou, J.G., Wang, X.L., Zhang, D. (2020). Cost analysis of hydrogen from the perspective of the whole industrial chain of production, storage, transportation and refueling. Natural Gas Chemical Industry, 45(1): 85-90, 96. https://doi.org/10.3969/j.issn.1001-9219.2020.01.018

[12] Dai, J.X., Zhang, Y.X. (2020). Safety analysis of hydrogen refueling station design. China Plant Engineering, (14): 238-240.

[13] Shi, J.H., Chang, Y.J., Khan, F., Zhu, Y., Chen, G.M. (2020). Methodological improvements in the risk analysis of an urban hydrogen fueling station. Journal of Cleaner Production, 257: 120545. https://doi.org/10.1016/j.jclepro.2020.120545

[14] Zheng, J.Y. (2020). High safety, low cost, large capacity storage of high pressure gaseous hydrogen. Journal of Zhejiang University (Engineering Science), 54(9): 16551657.

[15] Zheng, J.Y., Hu, J., Han, W.L., Hua, Z.L., Ye, S. (2020). Risk analysis and some countermeasures of pressure equipment for hydrogen energy in China. Pressure Vessel Technology, 37(6): 39-47.

[16] Agll, A.A.A., Hamad, T.A., Hamad, Y.M., Bapat, S.G., Sheffield, J.W. (2016). Development of design a drop-in hydrogen fueling station to support the early market buildout of hydrogen infrastructure. International Journal of Hydrogen Energy, 41(10): 5284-5295. https://doi.org/10.1016/j.ijhydene.2016.01.138

[17] Liu, F. (2020). Discussion on the whole-process project management of commercial hydrogen filling station. Energy Science and Technology, 10: 78-82.

[18] André, J., Auray, S., De Wolf, D., Memmah, M.M., Simonnet, A. (2014). Time development of new hydrogen transmission pipeline networks for France. International Journal of Hydrogen Energy, 39(20): 10323-10337.

https://doi.org/10.1016/j.ijhydene.2014.04.190

[19] Ramea, K. (2019). An integrated quantitative-qualitative study to monitor the utilization and assess the perception of hydrogen fueling stations. International Journal of Hydrogen Energy, 44(33): 18225-18239. https://doi.org/10.1016/j.ijhydene.2019.05.053

[20] Chen, T.C. (2019). Recommandations on the site selection and layout of domestic hydrogen fueling stations. Shanghai Chemical Industry, 44(7): 23-27. https://doi.org/10.16759/j.cnki.issn.1004017x.2019.07.013

[21] Kelley, S., Krafft, A., Kuby, M., Lopez, O., Stotts, R., Liu, J. (2020). How early hydrogen fuel cell vehicle adopters geographically evaluate a network of refueling stations in California. Journal of Transport Geography, 89:

https://doi.org/10.1016/j.jtrangeo.2020.102897

[22] Thiel, D. (2020). A pricing-based location model for deploying a hydrogen fueling station network. International Journal of Hydrogen Energy, 45(46): 24174-24189.

https://doi.org/10.1016/j.ijhydene.2020.06.178

[23] Gao, D.H., Li, W.Y., Liao, Y., Wang, S.R. (2020). Development planning of hydrogen refuelling stations network based on hydrogen supply locations and road transportation network. Natural Gas and Oil, 38(4): 3642. 5539.2020.04.007

[24] Li, L., Manier, H., Manier, M.A. (2020). Integrated optimization model for hydrogen supply chain network design and hydrogen fueling station planning. Computers \& Chemical Engineering, 134: 106683. https://doi.org/10.1016/j.compchemeng.2019.106683

[25] He, C., Sun, H., Xu, Y., Lv, S. (2017). Hydrogen refueling station siting of expressway based on the optimization of hydrogen life cycle cost. International Journal of Hydrogen Energy, 42(26): 16313-16324. https://doi.org/10.1016/j.ijhydene.2017.05.073

[26] Sun, H.R. (2018). Life-cycle based optimization of hydrogen refueling station siting and sizing. Southwest Jiaotong University.

[27] Lin, R., Ye, Z., Guo, Z., Wu, B. (2020). Hydrogen station location optimization based on multiple data sources. International Journal of Hydrogen Energy, 45(17): 10270-10279.

https://doi.org/10.1016/j.ijhydene.2019.10.069

[28] Miralinaghi, M., Lou, Y., Keskin, B.B., Zarrinmehr, A., Shabanpour, R. (2017). Refueling station location problem with traffic deviation considering route choice and demand uncertainty. International Journal of Hydrogen Energy, 42(5): 3335-3351. https://doi.org/10.1016/j.ijhydene.2016.12.137

[29] Lewandowska-Śmierzchalska, J., Tarkowski, R., UliaszMisiak, B. (2018). Screening and ranking framework for underground hydrogen storage site selection in Poland. International Journal of Hydrogen Energy, 43(9): 44014414. https://doi.org/10.1016/j.ijhydene.2018.01.089

[30] Narayanamoorthy, S., Ramya, L., Baleanu, D., Kureethara, J.V., Annapoorani, V. (2019). Application of normal wiggly dual hesitant fuzzy sets to site selection for hydrogen underground storage. International Journal 
of Hydrogen Energy, 44(54): 28874-28892. https://doi.org/10.1016/j.ijhydene.2019.09.103

[31] Deveci, M. (2018). Site selection for hydrogen underground storage using interval type-2 hesitant fuzzy sets. International Journal of Hydrogen Energy, 43(19): 9353-9368. https://doi.org/10.1016/j.ijhydene.2018.03.127

[32] Yang, G., Jiang, Y. (2020). Siting and sizing of the hydrogen refueling stations with on-site water electrolysis hydrogen production based on robust regret. International Journal of Energy Research, 44(11): 83408361. https://doi.org/10.1002/er.5440

[33] Grasman, S.E., Qin, R., Martin, K.B. (2013). An inventory modeling analysis of hydrogen fueling station capacity considering an outside option. Energy Systems, 4(2): 195-217. https://doi.org/10.1007/s12667-0120074-9

[34] Nowatzki, T., Ferris, M., Sankaralingam, K., Estan, C., Vaish, N., Wood, D. (2013). Optimization and mathematical modeling in computer architecture. Synthesis Lectures on Computer Architecture, 8(4): 1144. https://doi.org/10.2200/S00531ED1V01Y201308CAC0 26

[35] Grimm V., Schewe L., Schmidt M., Zöttl G. (2017). Uniqueness of market equilibrium on a network: A peakload pricing approach. European Journal of Operational Research, 261(3): 971-983 https://doi.org/10.1016/j.ejor.2017.03.036

[36] Siddiqui, S., Christensen, A. (2016). Determining energy and climate market policy using multiobjective programs with equilibrium constraints. Energy, 94: 316-325. https://doi.org/10.1016/j.energy.2015.11.002

[37] Ramos, M. A., Rocafull, M., Boix, M., Aussel, D., Montastruc, L., Domenech, S. (2018). Utility network optimization in eco-industrial parks by a multi-leader follower game methodology. Computers \& Chemical Engineering, 112: 132-153. https://doi.org/10.1016/j.compchemeng.2018.01.024

[38] Outrata, J.V., Ferris, M.C., Červinka, M., Outrata, M. (2016). On Cournot-Nash-Walras equilibria and their computation. Set-Valued and Variational Analysis, 24(3) 387-402. https://doi.org/10.1007/s11228-016-0377-4

[39] Xu, M., Chen, A., Gao, Z. (2008). An improved originbased algorithm for solving the combined distribution and assignment problem. European Journal of Operational Research, 188(2): 354-369. https://doi.org/10.1016/j.ejor.2007.04.047

[40] Lam, W.H., Huang, H.J. (1992). A combined trip distribution and assignment model for multiple user classes. Transportation Research Part B: Methodological, 26(4): 275-287. https://doi.org/10.1016/01912615(92)90038-X

[41] Ryu, S., Chen, A., Xu, X., Choi, K. (2014). A dual approach for solving the combined distribution and assignment problem with link capacity constraints. Networks and Spatial Economics, 14(2): 245-270. https://doi.org/10.1007/s11067-013-9218-2

[42] Yao, J., Chen, A., Ryu, S., Shi, F. (2014). A general unconstrained optimization formulation for the combined distribution and assignment problem. Transportation Research Part B: Methodological, 59: 137-160. https://doi.org/10.1016/j.trb.2013.11.007 